\title{
Generation of Amplifiable Genome-Specific Oligonucleotide Probes and Libraries
}

BioTechniques 33:874-882 (October 2002)

\section{Ivan Brukner, Guy A. Tremblay, and Bruno Paquin Université de Montréal, Montréal, Québec, Canada}

\begin{abstract}
Here we describe a process for the generation of oligonucleotide libraries representative of a given nucleic acid. Starting from a random pool of DNA oligonucleotides, the technique selects only those that hybridize to the nucleic acid template. This selection yields a highly specific library that represents an oligonucleotide image of the chosen template. The novel quality of this approach is the generation of amplifiable oligonucleotide probes that are of unique length and are easily subjected to differential selection. Here we apply this technique to produce different genomic oligonucleotide libraries and show that these genomic oligonucleotide libraries do not cross-hybridize. Differential selection of these genomic oligonucleotide libraries produces oligonucleotides that can be used in the identification, characterization, and isolation of nucleic acids.
\end{abstract}

\section{INTRODUCTION}

A number of diagnostic methods that involve nucleic acid hybridization have arisen in recent years. Most of them are designed to provide qualitative information about the presence of a specific sequence motif in a complex analytical mixture of nucleic acids and use a detection system based on PCR and/or DNA chip hybridization technologies $(5,6,12,15,16)$. For these technologies, diagnostic oligonucleotides constitute an essential part of the detection system. These oligonucleotides are primarily chosen based on the sequence data of the nucleic acids to be detected. In spite of the power of hybridization to identify correctly a complementary strand, it does have certain limitations. For example, the difference in stability between a perfectly matched complement and a complement mismatched at only one base can be as little as $0.5^{\circ} \mathrm{C}(7)$, a fact that constitutes a fundamental limitation to the power of DNA hybridization for specific identification of a cognate strand. Therefore, the diagnostic power of any chosen oligonucleotide must be validated on an analytical mixture whose sequence context is not totally known. The problem of adequate probe selection remains time and labor consuming at a moment when the growing complexity of detection systems based on oligonucleotide technologies requires a fast selection of a large number of suitable 20- to 25-mers.

The mismatch-containing oligonucleotide probes have better power of discrimination between two sequence motifs having just one nucleotide difference (7). Also, better mismatch discrimination occurred with 9-mer probes containing an additional internal mismatch (9). Additional mismatched bases located in the $3^{\prime}$-terminal regions of the specific primers are a way of improving the switching characteristics of the primer extension reactions during SNP detection $(2,17)$. Therefore, differential selection of redundant oligonucleotide probes now offers the generation of mismatch-containing probes with enhanced discrimination power among similar sequence motifs.

A simple method for generating a large number of specific probes that could potentially improve the signal-tonoise ratio of nucleic acid detection systems would prove valuable. Rapid oligonucleotide library manipulations that facilitate the duplication, subtraction, and merging of libraries could be used for that purpose. Here we report the development of a method called the genomic oligonucleotide library (GOL). A random pool of DNA oligonucleotides is hybridized to a template genomic DNA. Thus, the GOL is an oligonucleotide reflection of the template DNA. The GOL is homogeneous in length and amplifiable by PCR, and as such can be maintained virtually forever. Rather than relying on a rational selection of probes based on sequence data, this method depends on a more pragmatic selection based on experimental signal-to-noise discrimination criteria. This makes the GOL method potentially useful for applications that require the rapid generation of a large number of oligonucleotide probes for nucleic acid detection. Using a random introduction of mismatches, the selected probes acquired an enhanced power of detection of small differences between sequence motifs. 


\section{MATERIALS AND METHODS}

\section{DNA/Oligonucleotides}

The starting random DNA pool (RAN) was synthesized by Invitrogen (Burlington, Ontario, Canada) (5'GCCTGTTGTGAGCCTCCTGTCGAA-N20-TTGAGCGTTTATTCTTGTCTCCC-3'). The corresponding left and right arms were LEFT (5'GCCTGTTGTGAGCCTCCTGTCGAA- $3^{\prime}$ ) and BioRIGHT (5'-BioGGGAGACAAGAATAAACGCTCAA-3'). The 5'-end biotinylated oligonucleotides were used to bind complementary strands, using BioMag ${ }^{\circledR}$ magnetic particles (PerSeptive Biosystems, Framingham, MA, USA). During preparative hybridization, the left and right arms were blocked by cLEFT (5'TTCGACAGGAGGCTCACAACAGGC-3') and RIGHT (5'-GGGAGACAAGAATAAACGCTCAA-3'). These oligonucleotides are termed "blockers" in the text. The following genomic DNAs were used to produce GOLs: Adenovirus DNA Type 2, (Invitrogen), $\lambda$ DNA cI857 ind1 Sam 7 (New England Biolabs, Beverly, MA, USA), pBluescript $^{\circledR}$ II SK ${ }^{+}$(Stratagene, San Diego, CA, USA). The human HeLa DNA we used in one of our controls is from BD Biosciences Clontech (Palo Alto, CA, USA).

\section{Blotting Genomic DNA}

The genomic DNA was denatured 2-3 min at $95^{\circ} \mathrm{C}$ and cooled on ice. Denatured genomic DNA (100 ng) was blotted on the nylon membrane (Hybond- ${ }^{\circledR}$; Amersham Biosciences, Piscataway, NJ, USA), dried for $2 \mathrm{~min}$ on a hot plate, and exposed to UV light for 8 $\mathrm{min}$. The prehybridization was done for a minimum of $30 \mathrm{~min}$ in the hybridization buffer (7\% SDS, $0.25 \mathrm{M} \mathrm{Na}_{2} \mathrm{HPO}_{4}$, pH 7.4, 1 mM EDTA, and 1\% BSA).

\section{Hybridization and Washing of the Starting Random Pool}

The preparative hybridization between random core $(20 \mathrm{~N})$ and targeted DNA was done with 10 pmol starting random pool (RAN). The random pool was pre-mixed with 100 pmol (10 times more than RAN) of LEFT and RIGHT blockers to exclude cross-hybridization of left and right arms with genomic DNA. The oligonucleotide mixture was heated at $95^{\circ} \mathrm{C}$, cooled down to room temperature, and added to the hybridization buffer. The hybridization was done overnight at $50^{\circ} \mathrm{C}$. The membranes were washed several times, first in $6 \times$ SSC and then in $2 \times$ SSC, at the same temperature as the hybridization was done.

\section{Generating Oligonucleotide Libraries by PCR}

The dot containing the genomic DNA and bound probes was cut out of the nylon membrane (radius of 2-4 $\mathrm{mm}$ ), soaked in $100 \mu \mathrm{L}$ water, and heated to $95^{\circ} \mathrm{C}$ for $1-2 \mathrm{~min}$. The solution containing the denatured probe (originally RAN) was then collected and passed through a Sephadex ${ }^{\circledR}$ G-50 (Amersham Biosciences) column to eliminate salts and SDS. The PCR was prepared under standard conditions, typical for SELEX-like amplification of DNA $(4,13)$. The RIGHT $5^{\prime}$-end biotinylated primer of the sense strand (the one that did not hybridize with genomic DNA) and LEFT primer of the antisense strand were used in the reactions. The PCR conditions were $20 \mathrm{cy}$ cles of denaturation at $95^{\circ} \mathrm{C}$ for $30 \mathrm{~s}$, annealing at $53^{\circ} \mathrm{C}$ for $30 \mathrm{~s}$, and elongation at $72^{\circ} \mathrm{C}$ for $30 \mathrm{~s}$.

\section{Probe Labeling and Hybridization}

Before labeling, the PCR mixtures were passed through Sephadex G-50 columns. PCR product (100-200 ng) was labeled with 50 pmol $\gamma\left[{ }^{32} \mathrm{P}\right]$ ATP $(6000 \mathrm{Ci} / \mathrm{mmol}$; I.C.N. Pharmaceuticals, Irvine, CA, USA). The total amount of probe radioactivity was $300000 \mathrm{cpm}$. The probe was added to $0.5 \mathrm{~mL}$ hybridization buffer. The blotting of genomic DNA was done as described above. Hybridizations were done overnight at $50^{\circ} \mathrm{C}$. The nylon membranes were washed as described above and exposed onto $\operatorname{Kodak}^{\circledR} \mathrm{X}^{\circledR}$-Omat ${ }^{\circledR}$ film (Eastman Kodak, Rochester, NY, USA).

\section{GOL Labeling and Analytical Hybridization}

The generated GOLs are tested using $(i)$ the original genomic DNA from 
which they were selected (positive control) and (ii) using the unrelated genomic DNA (negative control). The GOL labeling, hybridization, and probe washing were done as described, except that the hybridization time was shorter (60 min).

\section{Southern Blot Hybridization}

Electrophoresis was performed in a $1 \%$ agarose gel with TBE buffer (80 $\mathrm{mM}$ Tris adjusted to $\mathrm{pH} 8.0$ with boric acid, $2 \mathrm{mM}$ EDTA) and stained with ethidium bromide. One microgram of $B s t$ EII-digested $\lambda$ DNA, 300 ng adenoviral DNA, and $1 \mu \mathrm{g}$ AluI-HpaI-digested human HeLa DNA were run on the gel according to specifications (all restriction enzymes used in this work were purchased from New England Biolabs). For Southern hybridization, DNA was transferred to nylon membranes by capillary blot procedure following manufacturer's recommendations (Amersham Biosciences). Hybridization was performed as described above with adenoviral GOL. Autoradiographic exposure (using $\mathrm{X}$-Omat ${ }^{\circledR}$ film) was done at room temperature for a few hours. Stripping was achieved by pouring a boiling $1 \%$ SDS solution over the nylon membrane.

\section{Subtractive Enrichment of GOL}

The tester GOL (mixed GOL) was prepared from equimolar mixtures of the two genomes (adenovirus type 2 and $\lambda$ ). The driver GOL was produced from the $\lambda$ genome only. The production of sense strand (the one that did not hybridize with genomic DNA) was done using 5 -end biotinylated primer in PCR. After denaturing the PCR product, the biotinylated sense strand was bound to streptavidin magnetic particles $(200 \mu \mathrm{g}$, binding capacity greater than 200 pmol biotinylated oligonucleotides; BioMag magnetic particles) and bound using a magnet. The complementary antisense strand was discarded with the liquid phase. The mixed antisense tester GOL ( $\lambda$ and adenovirus DNA) was produced in the same way. This time, the supernatant with the antisense, non-biotinylated strand was hybridized overnight at $50^{\circ} \mathrm{C}$ with 10 times molar excess of dri- ver $\lambda$ sense strand attached to the magnetic beads. The hybridization buffer was the same as described above but without SDS. After removing the fraction bound to the magnetic beads, the rest of the mixture was used in the analytical hybridization step.

\section{RESULTS}

\section{Initial Complexity of GOL Probes}

The starting random pool of oligonucleotides contains $4^{20}$ (i.e., $10^{12}$ ) different 20-mers. Such a mixture contains a pool of $10^{11}$ extra sequence motifs, which are not present in the human genome $\left(10^{12}-10^{9}>10^{11}\right)$. This pool of oligonucleotides is used to generate mismatch-containing probes with the better specificity/discrimination power over classical probes. One should note that if the length of the random sequence motif $(\mathrm{N})$ were longer, then a higher number of mismatch-containing motifs would exist in a GOL. Increasing the length of the random sequence motif from $20 \mathrm{~N}$ to $25 \mathrm{~N}$ would produce $10^{15}$ extra sequence motifs, which could not find their full-match complementary sequences, even in the most complex genomes. However, the increase in the length of $\mathrm{N}$ would allow the generation of additional mismatch/match combinations. That leads to better discrimination between two sequence motifs that differ by only one nucleotide. Therefore, the discrimination power of detection (specificity) will augment with the length of random motif of GOL oligonucleotides. A procedure for generating GOLs is described in detail in the Materials and Methods section.

We used blockers to avoid hybridization of the flanking arms to the targeted genome, and this step was found to be critical to achieve specificity (WO/0043538; Université de Montréal). The stringency of hybridization conditions eliminates unbound 20mers, leaving the specific oligonucleotides bound to the membrane via hybridization of the random core to the genome. This ensemble of selected oligonucleotides constitutes the GOL.

One should note that the starting random pool of oligonucleotides (1020 pmol) contained about eight copies of each sequence motif during the first hybridization step. The number of copies of each particular 20-mer present in the random mixture was smaller than the number of genome copies used in this procedure.

\section{Specificity and Distribution of GOL Probes}

Figure 1 shows that GOLs are able to discriminate genomes with complexities of $10^{3}-10^{4}$. The starting random pool of probes binds to all three genomes equally (Figure 1, row 1). After one round of selection, the GOL can hybridize specifically to a single targeted genome (Figure 1, rows 2, 3, and 4). The GOL can also be selected against a mixture of two genomes, and the specificity is conserved for both genomes (Figure 1, row 5).

A Southern blot was performed to document the distribution of adenovirus GOL probes along the genome (Figure 2). There was no apparent cross-hybridization of adenovirus GOL to either HeLa or $\lambda$ DNA (Figure $2 b$, lanes 1,4 , 5 , and 6). The intensity of radioactive signal over adenoviral genome generated by adenovirus-specific GOL was linearly increasing with the DNA fragments' length (Figure 3). Therefore, one could deduce a uniform distribution of GOL throughout the genomic DNA.

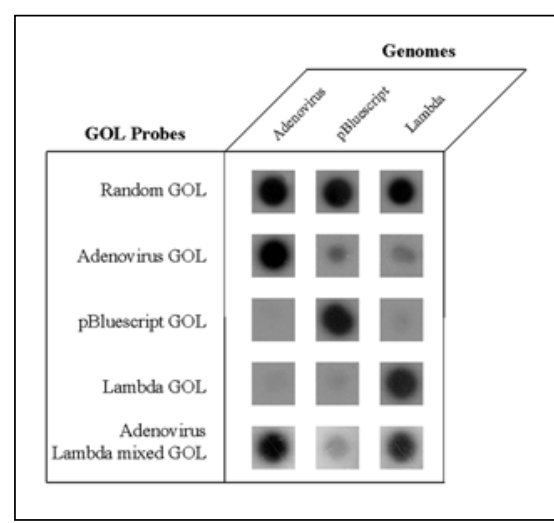

Figure 1. Dot blot hybridization of GOL targeted against different genomes. The first row represents the dot blot hybridization of random probes with the specified genomic DNA (adenovirus, pBluescript, and $\lambda$ ). The other rows are analytical dot blot hybridizations of selected GOLs with the corresponding genomes. The last row shows dot blot hybridization of mixed adenovirus and $\lambda$-selected GOLs. The procedures of preparative and analytical hybridization are described in the Materials and Methods section. 
We then selected only the subset of the adenovirus GOL bound to the 3648bp band, by cutting the membrane and extracting the DNA as described above. We performed another Southern analysis using the GOL subset as a probe against the whole adenovirus genome, and we observed a specific hybridization to the 3648-bp band (Figure 2 and Figure 3, B and C). These data illustrate that we were able to increase the specificity and reduce the complexity of the original adenovirus-specific GOL to that of the 3648-bp subset, using just one additional round of selection.

\section{Subtractive Enrichment of GOLs}

One round of subtractive enrichment between two GOLs was performed. The tester GOL reflects the two genomes (adenovirus type 2 and $\lambda$ phage), whereas the driver GOL reflects only the $\lambda$ genome. The singlestranded GOL from the driver DNA was used to bind the complementary single-stranded mixed tester GOL. After removing the subtracted fraction, the remaining ssDNA was used as a probe in the analytical hybridization step. The hybridization signals between the $\lambda$ and adenovirus genomes before (Figure 4A) and after (Figure 4B) one round of subtractive enrichment is shown for illustrative purposes. The subtraction was done to show the potential use of GOL technology in generating differential probes. Only one cycle of subtraction was performed, and background was decreased but not removed. Additional subtraction steps should remove background (8).

\section{Complexity of Selected GOL Probes}

In Table 1, we predicted the relative distribution of 20-mers with different numbers of matches that hybridized to the targeted DNA. We calculated the number of 20-mers with $\mathrm{k}$ number of matches, capable of hybridizing to the target sequence. We first calculated the number of combinations of 20-mers $\left(\mathrm{C}_{\mathrm{k}}{ }^{20}\right)$ with the same number of matches $(\mathrm{k})$ in the initial random pool of oligonucleotides that are capable of hybridizing to the specific 20 -mer motif. Since each full match could be replaced

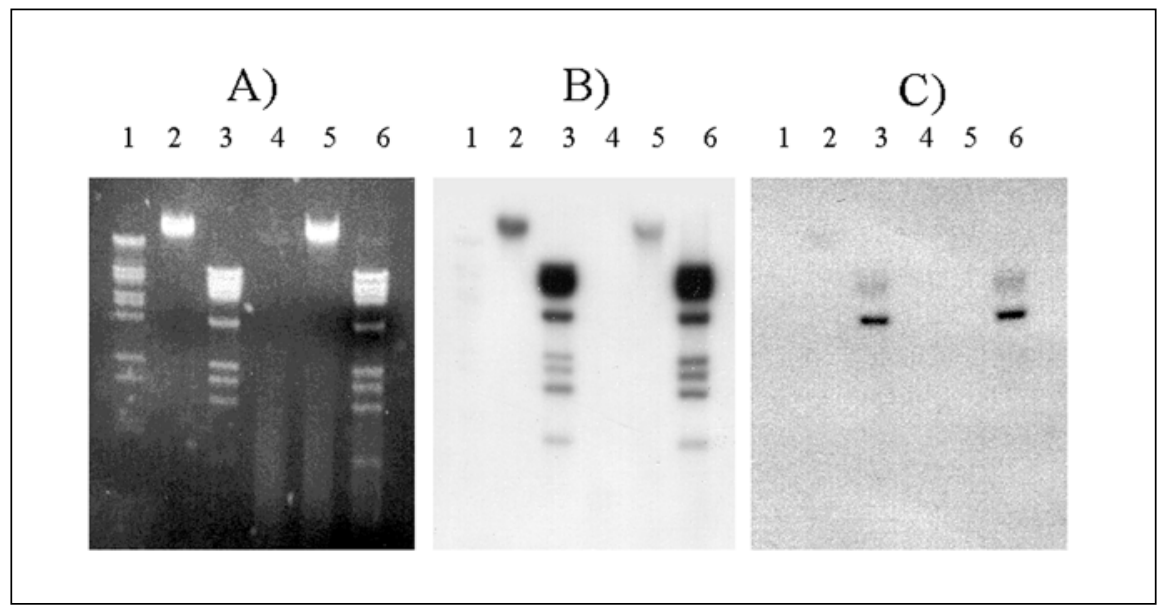

Figure 2. Specificity and probe distribution of GOL generated from the adenoviral genome. (A) The corresponding genome and adenoviral DNA were run on a $1 \%$ agarose gel stained with ethidium bromide. The type of restriction enzyme and DNA are indicated on the top of each gel lane: $1, \lambda$ BstEII; 2, adenovirus; 3, adenovirus KpnI; 4, HeLa DNA AluI + HpaI; 5, adenovirus and HeLa DNA AluI + $H p a \mathrm{I}$; 6, adenovirus KpnI and HeLa DNA AluI + HpaI. (B) Southern hybridization of the same gel using adenovirus GOL as a hybridization probe (see Figure 1, row 2). Note that under those conditions, there was no cross-hybridization with either $\lambda$ or human DNA. (C) The same membrane was stripped and rehybridized with a GOL directed against a 3648-bp restriction fragment. This subset of adenovirus GOL was prepared by cutting the membrane corresponding to the 3648-bp band from a similar Southern blot and re-amplified by PCR as described in the Materials and Methods section. Only those members of adenoviral GOL that were selected from the particular adenoviral restriction fragment (3645 bp) hybridized back to the same fragment and only to the fragment from which they were selected. Obviously, these GOL probes are not promiscuously hybridizing to other fragments of the adenoviral genome. Thus, we show that GOL specificity could be additionally increased by controlling the choice of targeted DNA fragments in the next round of selection.

by three different mismatches $(\mathrm{m}=20$ $\mathrm{k}$ ), the number of combinations $\mathrm{C}_{\mathrm{k}}{ }^{20}$ must be multiplied by the number of sequence variations, $3^{\mathrm{m}}$, for each combination. Finally, we adjusted these numbers to reflect the sequence-dependent thermostability of 20 -mers with mismatches (14) that hybridized at $50^{\circ} \mathrm{C}$ using the thermostable fraction of the binomial distribution for each kmer population. Duplexes of more than eight mismatches are not observed based on this thermostability criterion.

\section{DISCUSSION}

We have described a new method that generates amplifiable DNA oligonucleotide libraries that are specific for a given segment of DNA. This method is reminiscent of the SELEX method $(4,13)$ in that we use amplification arms to amplify specific oligonucleotides, selected from an initial random pool. It could also be compared to random priming because we are capable of generating probes without a priori knowledge of the template sequence. One round of preparative hybridization was enough to produce GOLs (Figures 1 and 2). The GOLs were inferred from genomes of complexity of $10^{3}-10^{4}$. According to our calculations, we expect that the 20-mers selected in a GOL can contain up to eight mismatches. Nevertheless, we were able to achieve specificity toward a given template. As reported before in the literature $(2,7$, $9,17)$, the mismatch-free hybridization is not the critical factor for the differential detection approach, but rather relative difference in the thermodynamic stability between hybridized oligonucleotides. In fact, selected mismatchcontaining oligonucleotides do have enhanced power of detection over mismatch-free oligonucleotides $(2,7,9,17)$.

This technique uses the selection of oligonucleotides based on a pragmatic criterion and allows the possibility of overcoming the limitations of current techniques. For example, the differential melting temperatures of each probe/analyte hybrid present a technological obstacle for multiplex SNP detection by hybridization, using DNA/ RNA probes bound to the solid support. The present method offers a solution to 
Table 1. The Relative Distribution of Random 20-Mers with Different Numbers of Matches that Hybridized to the Target at $50^{\circ} \mathrm{C}$

\begin{tabular}{|c|c|c|c|c|c|}
\hline $\begin{array}{l}\text { Number of Pairing } \\
\text { Bases in a Random } \\
\text { 20-Mer Hybridized } \\
\text { to Template DNA (k) }\end{array}$ & $\begin{array}{c}\text { Number of } \\
\text { Combinations } \\
\text { of } k \text { in a 20-Mer } \\
\left(C_{k}{ }^{20}\right)\end{array}$ & $\begin{array}{c}\text { Number of } \\
\text { Sequence } \\
\text { Variations for } \\
\text { Each } C_{k}{ }^{20}\left(3^{20-k}\right)\end{array}$ & $\begin{array}{l}\text { Fraction of the } \\
20 \text {-Mers with a } \\
T_{m} \text { over } 50^{\circ} \mathrm{C} \\
\text { (f) }\end{array}$ & $\begin{array}{l}\text { Total number of } \\
20 \text {-Mers with a } \\
T_{m} \text { over } 50^{\circ} \mathrm{C} \\
\left(C_{k}^{20}\right)\left(3^{20-k}\right)(f)\end{array}$ & $\begin{array}{c}\text { Relative } \% \text { of } 20 \text {-Mers } \\
\text { that Hybridized } \\
\text { to Template DNA } \\
\left(C_{k}{ }^{20}\right)\left(3^{20-k}\right)(f)\end{array}$ \\
\hline 11 & $1.7 \times 10^{5}$ & $2.0 \times 10^{4}$ & 0.00 & 0 & 0 \\
\hline 12 & $1.2 \times 10^{5}$ & 6561 & 0.02 & $1.7 \times 10^{7}$ & 35.43 \\
\hline 13 & $7.8 \times 10^{4}$ & 2187 & 0.13 & $2.2 \times 10^{7}$ & 47.25 \\
\hline 14 & $3.9 \times 10^{4}$ & 729 & 0.21 & $5.9 \times 10^{6}$ & 12.72 \\
\hline 15 & $1.6 \times 10^{4}$ & 243 & 0.50 & $1.9 \times 10^{6}$ & 4.04 \\
\hline 16 & 4845 & 81 & 0.60 & $2.4 \times 10^{5}$ & 0.50 \\
\hline 17 & 1140 & 27 & 0.83 & $2.6 \times 10^{4}$ & 0.05 \\
\hline 18 & 190 & 9 & 0.88 & 1504 & 0.0032 \\
\hline 19 & 20 & 3 & 0.97 & 58 & 0.0001 \\
\hline 20 & 1 & 1 & 0.99 & 1 & 0.000002 \\
\hline \multicolumn{6}{|c|}{$\begin{array}{l}\text { Fraction of the } 20 \text {-mers with a } T_{m} \text { over } 50^{\circ} \mathrm{C} \text { was calculated using the Wallace rule (14), which is applicable for short se- } \\
\text { quences that have } 20 \text { bases or less, using a first-order approximation: } T_{m}=2^{\circ} \mathrm{C}(A+T)+4^{\circ} \mathrm{C}(\mathrm{G}+\mathrm{C}) \text {. This equation assumes } \\
\text { a salt concentration of } 0.9 \mathrm{M} \text {, typical of dot blot and other hybridization assays. }\end{array}$} \\
\hline
\end{tabular}

this problem. The major use of this technique would be in building simple but reliable DNA/RNA diagnostic devices with enhanced power of identification of single nucleotide differences over low-complexity analyte (i.e., DNA or RNA products that hybridize with selected probes). This includes the genotyping of clinically relevant, drugresistant mutations of human immun-

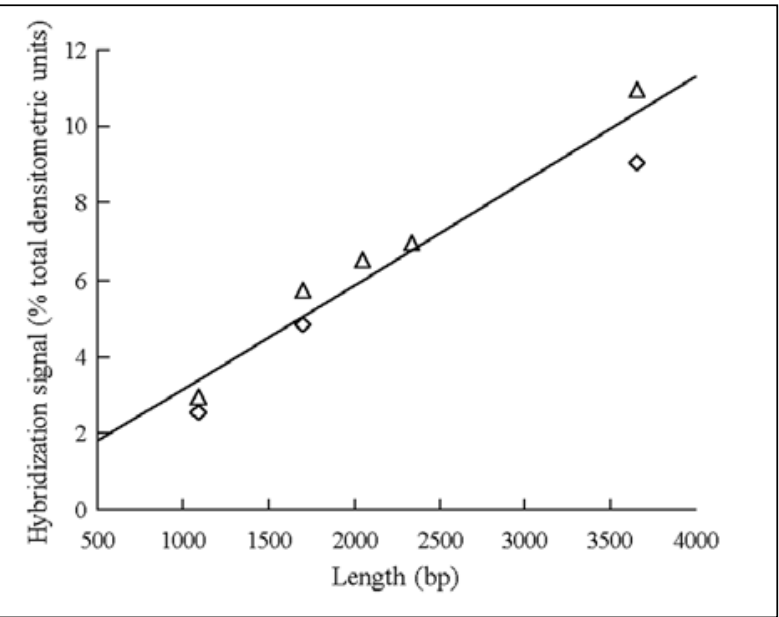

Figure 3. The distribution of GOL along genomic DNA. The densitometric scan of radioactive signal from GOL was integrated over total adenoviral genome (see Figure 2, lanes 3 and 5) using Scion Image software (Scion, Frederick, MD, USA). The signal intensity of GOL probes hybridizing to restriction fragments is linearly proportional to the length of DNA. get. They are also reminiscent of those of Mishra et al. (11) and Ducongé et al. (3), who use in vitro selection methods to obtain high-affinity and specific antisense oligonucleotides. Although the strategies of preparative hybridization are similar, the uses and applications are very different. Our procedure results in the generation and selection of oligonucleotide probes with a high specificity for a given system. These oligonucleotides cover the target DNA, thus increasing detectability, which might be lost in classical oligonucleotide detection systems because of et al. (1) in that we are able to recover and amplify DNA probes hybridized to an immobilized tar-

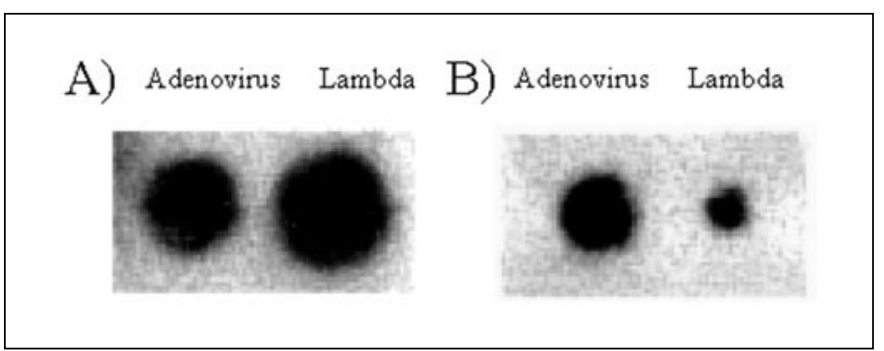

Figure 4. Subtractive enrichment of GOL. The tester GOL was represented by the mixture of two genomes (adenovirus type 2 and $\lambda$ phage). The driver GOL was produced from the $\lambda$ genome only. The single-stranded GOL from driver DNA was used to pool out the complementary single-stranded mixed tester GOL. After removing the subtracted fraction, the rest of mixed GOL was used as a probe in the analytical hybridization step. The mixed GOL probes were analyzed by dot blotting as described before (A) and after (B) subtractive enrichment and by hybridization to genomic adenovirus and $\lambda$ DNA. 
the DNA secondary structure or DNA deletion in the analytical mixture. Recent excellent achievements in the field of subtractive hybridization $(8,10)$ are offering procedures that are fully compatible with the design of our GOLs. The potential advantage of working with GOL versus other nucleic acid libraries (cDNA) is that the length of the GOL is uniform and the libraries are self-amplifiable and easily subjected to subtraction. Selected oligonucleotides, obtained by our approach, are also compatible with DNA array technologies. Recently, Sigma-Genosys (The Woodlands, TX, USA) put on the market new product OligoLibraries ${ }^{\mathrm{TM}}$ that incorporates multiple oligonucleotides/probes per gene and maximizes the detection potential. This strategy consists of a computer-based selection of probes, enabling one to address redundancy, to incorporate alternative splicing, and to consider specificity and cross-homology in the design of optimal oligonucleotides for gene expression, drug discovery, or functional assays. Differences in GC content and/or melting temperature are normalized across the oligonucleotide collection to ensure identical hybridization conditions. Our GOL technology solves the same problems, but it is based on the sequence-de- pendent selection of oligonucleotides with the best signal-to-noise ratio. The advantage of GOL technology is that it is not dependent on the present content of genomic databases and/or predictive computer-assisted oligonucleotide selection rules. Therefore, GOL can be the best choice for the next generation of DNA diagnostics.

\section{ACKNOWLEDGMENTS}

We thank Pierre Chartrand, Thomas Hudson, and Larry Malek for helpful comments and trust, and Alice Rae for critical reading of the manuscript. Parts of the data presented in this manuscript are described in the patent $\mathrm{WO} /$ 0043538 (Université de Montréal). We are also profoundly grateful to Robert Cedergren, who shared a vision and allowed us to work together on this project. This work was supported by operating grants from the Medical Research Council (Canada) and the Natural Sciences and Engineering Research Council (Canada) to R.C.

\section{REFERENCES}

1.Armour, J.A., C. Sismani, P.C. Patsalis, and G. Cross. 2000. Measurement of locus copy number by hybridisation with amplifiable probes. Nucleic Acids Res. 28:605-609.

2.De Milito, A., M. Catucci, F. Iannelli, L. Romano, M. Zazzi, and P.E. Valensin. 1995. Increased reliability of selective PCR by using additionally mutated primers and a commercial Taq DNA polymerase enhancer. Mol. Biotechnol. 2:166-169.

3.Ducongé, F. and J.J. Toulme. 1999. In vitro selection identifies key determinants for looploop interactions: RNA aptamers selective for the TAR RNA element of HIV-1. RNA 5:1605-1614.

4.Fitzwater, T. and B. Polsky. 1996. A SELEX primer. Methods Enzymol. 267:275-301.

5.Fredricks, D.N. and D.A. Relman. 1999. Localization of Tropheryma whippelii rRNA in tissues from patients with Whipple's disease. Clin. Infect. Dis. 29:475-486.

6.Gerhold, D., T. Rushmore, and C.T. Caskey. 1999. DNA chips: promising toys have become powerful tools. Trends Biochem. Sci. 24:168-173.

7.Guo, Z., Q. Lui, and L.M. Smith. 1997. Enhanced discrimination of single nucleotide polymorphisms by artificial mismatch hybridization. Nat. Biotechnol. 15:331-335.

8.Luo, J.H., J.A. Puc, E.D. Slosberg, Y. Yao, J.N. Bruce, T.C. Wrigh0,t Jr., M.J. Becich, and R. Parsons. 1999. Differential subtraction chain, a method for identifying differ- ences in genomic DNA and mRNA. Nucleic Acids Res. 27:e24.

9.Maldonado-Rodriguez, R., M. EspinosaLara, P. Loyola-Abitia, W.G. Beattie, and K.L. Beattie. 1999. Mutation detection by stacking hybridization on genosensor arrays. Mol. Biotechnol. 1:13-25.

10.Matz, M.V. and S.A. Lukyanov. 1998. Different strategies of differential display: areas of application. Nucleic Acids Res. 26:55375543.

11.Mishra, R.K., R. Le Tinevez, and J.J. Toulme. 1996. Targeting nucleic acid secondary structures by antisense oligonucleotides designed through in vitro selection. Proc. Natl. Acad. Sci. USA 93:10679-10684.

12.Shoemaker, D.D., E.E. Schadt, C.D. Armour, Y.D. He, P. Garrett-Engele, P.D. McDonagh, P.M. Loerch, A. Leonardson, et al. 2001. Experimental annotation of the human genome using microarray technology. Nature 409:922-927.

13.Singer, B.S., T. Shtatland, D. Brown, and L. Gold. 1997. Libraries of genomics SELEX. Nucleic Acids Res. 25:781-786.

14.Wallace, R.B., J. Shaffer, R.F. Murphy, J. Bonner, T. Hirose, and K. Itakura. 1979. Hybridization of synthetic oligodeoxyribonucleotides to phi chi 174 DNA: the effect of single base pair mismatch. Nucleic Acids Res. 6:3543-3547.

15.Watson, A., A. Mazumder, M. Stewart, and S. Balasubramanian. 1998. Technology for microarray analysis of gene expression. Curr. Opin. Biotechnol. 9:609-614.

16.Whitcombe, D., C.R. Newton, and S. Little. 1998. Advances in approaches to DNA-based diagnostics. Curr. Opin. Biotechnol. 9:602608.

17.Zhou, G., M. Kamahori, K. Okano, G. Chuan, K. Harada, and H. Kambara. 2001. Quantitative detection of single nucleotide polymorphisms for a pooled sample by a bioluminometric assay coupled with modified primer extension reactions (BAMPER). Nucleic Acids Res. 19:E93.

Received 3 April 2002; accepted 10 June 2002.

Address correspondence to:

Dr. Ivan Brukner

3754 Kent

Montreal, QC, H3S 1N3, Canada

e-mail:ibrukner@hotmail.com

For reprints of this or any other article, contact Reprints@BioTechniques.com 